\title{
Propriedades termodinâmicas da adsorção de água de grãos de Coffea arabica L.
}

\section{Thermodynamic properties of water adsorption of Coffea arabica L. grains}

\author{
Samuel Gonçalves Ferreira dos SANTOS ${ }^{1}$; Vinicius Gonçalves ALMEIDA²; Dennis Ricardo Cabral CRUZ³; \\ Renato Souza RODOVALHO ${ }^{4}$ \\ ${ }^{1}$ Autor para correspondência, Engenheiro Agrônomo, Instituto Federal Goiano - Campus Ceres, Instituto Federal Goiano - \\ Campus Ceres, samuel-2100@hotmail.com \\ ${ }^{2}$ Engenheiro Agrônomo, Instituto Federal Goiano - Campus Ceres, Instituto Federal Goiano -Campus Ceres, \\ almeidaagropec@gmail.com \\ ${ }^{3}$ Mestrando em Produção Vegetal, Universidade Federal de Goiás, Universidade Federal de Goiás, denisribral@gmail.com \\ ${ }^{4}$ Doutor em Agronomia, IF Goiano - Campus Ceres, renato.rodovalho@ifgoiano.edu.br
}

Recebido em: 02-06-2020; Aceito em: 27-10-2020

\begin{abstract}
Resumo
Os grãos de café (Coffea spp.) apresentam longevidade altamente dependente da disponibilidade de água em seu interior, sendo que o incremento do teor de água das mesmas pode prejudicar o armazenamento devido ao provável aparecimento de microrganismos. O estudo das propriedades termodinâmicas proporciona o conhecimento da afinidade do sorvente pela água e da espontaneidade do processo de sorção, sendo parâmetros de suma importância no processo de secagem, na análise de projetos de equipamentos, processos de preservação e acondicionamento de grãos. Objetivou-se, com o presente trabalho, determinar e avaliar as propriedades termodinâmicas em grãos de Coffea arabica, com teores de água de equilíbrio na faixa de 1,91 a 25,34\% base seca (b.s.) e atividade de água entre 0,10 a 0,83 (decimal), nas temperaturas de $30,40,50$ e $60^{\circ} \mathrm{C}$. O calor latente de vaporização, a entropia diferencial e a energia livre de Gibbs aumentam com a redução do teor de água dos grãos. A temperatura isocinética de $498,3433 \pm 5,7013 \mathrm{~K}$ confirma a compensação química linear entre a entalpia e a entropia diferenciais dos grãos de Coffea arabica, sendo o processo de adsorção dos grãos controlado pela entalpia. A adsorção da água nos grãos de Coffea arabica é um processo não espontâneo.
\end{abstract}

Palavras-chave adicionais: energia livre de Gibbs; entropia; teoria isocinética.

\begin{abstract}
The coffee beans (Coffea spp.) Have longevity highly dependent on the availability of water in their interior, and the increase in their moisture content may impair storage due to the likely appearance of microorganisms. The study of thermodynamic properties provides knowledge of the affinity of the sorbent for water and the spontaneity of the sorption process, being parameters of paramount importance in the drying process, in the analysis of equipment designs, preservation processes and grain conditioning. The objective of this work was to determine and evaluate the thermodynamic properties in Coffea arabica grains, with equilibrium water contents in the range of 1.91 to $25.34 \%$ dry basis (d.b) and water activity between 0.10 to 0.83 (decimal), at temperatures of $30,40,50$ and $60^{\circ} \mathrm{C}$. The latent heat of vaporization, the differential entropy and the free Gibbs free energy increase with the reduction of the moisture content of the grains. The isokinetic temperature of $498.3433 \pm 5.7013 \mathrm{~K}$ confirms the linear chemical compensation between enthalpy and differential entropy of Coffea arabica grains, with the grain adsorption process being controlled by enthalpy. The adsorption of water on Coffea arabica grains is a non-spontaneous process.
\end{abstract}

Additional keywords: entroy; Gibbs free energy; isokinetic theory.

\section{Introdução}

O consumo interno de café no Brasil chegou a 21 milhões de sacas, no período de novembro de 2017 a outubro de 2018. Esses números elevaram o consume per capita para $6,02 \mathrm{~kg} / \mathrm{ano}$ de café cru e $4,82 \mathrm{~kg} /$ ano de café torrado e moído, o que mantém o Brasil como o segundo maior consumidor de café do mundo. (ABIC, 2018). Todavia, para que o Brasil consiga se consolidar como um grande produtor/consumidor de café é necessário a realização de pesquisas relacionadas a pós-colheita deste produto.

Os grãos de café (Coffea spp.) apresentam longevidade altamente dependente da disponibilidade de água em seu interior, sendo que o incremento do teor de água das mesmas pode prejudicar 0 armazenamento devido ao aparecimento de microrganismos (Bakhtavar \& Basra, 2019). No Brasil, destacam-se duas espécies do gênero Coffea, Coffea arabica L. (café arabica) e o Coffea canephora Pierre ex Froehner (café robusta, também conhecido como café conilon ou canelão) sendo que a primeira corresponde 
a aproximadamente $75 \%$ do total produzido (Corrêa et al., 2014).

O conhecimento relacionado a higroscopicidade consiste em um fator chave nos projetos e estudos de secagem, manuseio, armazenamento, embalagem e sistemas de transporte e na longevidade de sementes, grãos e outros produtos agrícolas (Araújo et al.,2001). Dessa forma, as isotermas de sorção consistem em modelos matemáticos que são capazes de expressar a relação entre 0 teor de água de um determinado produto e a atividade de água para uma temperatura específica (Costa et al., 2015), sendo de extrema importância no fornecimento de informações relacionadas ao equilíbrio higroscópico, que podem ser utilizadas diretamente nos processos de secagem e armazenamento (Ferreira Junior et al., 2018).

Além de serem utilizadas para prever a estabilidade dos alimentos durante $\mathrm{o}$ armazenamento, facilitando assim a escolha de materiais de embalagem apropriados (Thys et al., 2010), as isotermas de sorção fornecem informações relacionada as propriedades termodinâmicas dos alimentos. Estas quantificam os valores de entalpias, entropias e, também, a energia livre de Gibbs que ocorrem durante o processo de sorção dá água nos alimentos (Kaya \& Kahyaoglu, 2006).

O calor latente da vaporização consiste na quantidade de energia necessária para evaporar a água do produto (Corrêa et al., 1998). Entalpia é uma indicação da força da água interconexão de moléculas de vapor com os locais de sorção ativos (Oliveira et al., 2014) e a entropia está associada ao grau de desordem das moléculas de água (Majd et al., 2013). Já a energia livre de Gibbs avalia a afinidade entre o produto e a água, podendo indicar se a saída de água do produto, durante o processo de secagem, é espontânea ou não (Oliveira et al., 2013).

Diversos estudos já foram realizados com o intuito de determinar os parâmetros termodinâmicos como o calor latente de vaporização, entalpia e entropia dentre outros, para produtos como sementes de ryegrass (Lolium multiflorum L.) (Zeymer et al., 2020), sementes de Buchenavia capitata (Vahl) Eichler (Silva et al., 2018) e sementes de pimenta Cumari-do-Pará (Rodrigues et al., 2020) e grãos de milho (Oliveira et al., 2013). Todavia, não há muitos estudos referente as propriedades termodinâmicas de adsorção em grãos de Coffea arabica.

Neste contexto, objetivou-se, com o presente trabalho, determinar e avaliar as propriedades termodinâmicas em grãos de Coffea arabica, com teores de água de equilíbrio na faixa de 1,91 a 25,34\% base seca (b.s.) e atividade de água entre 0,10 a 0,83 (decimal), nas temperaturas de $30,40,50$ e $60^{\circ} \mathrm{C}$.

\section{Material e métodos}

O estudo foi desenvolvido no Laboratório de Química Instrumental, do Instituto Federal GoianoCampus Ceres, no município de Ceres, estado de Goiás, durante o dia 02 de setembro de 2017 até o dia 10 de fevereiro de 2018. Foram utilizados grãos de Coffea arabica L., da cultivar Acauã Novo, colhidas na própria Instituição (Safra 2017/18), cujas coordenadas cartesianas são: UTM: $E=649.582,00 \mathrm{~m}$ e $\mathrm{N}=$ 8.302.194,00 m; altitude de $556 \mathrm{~m}$, com clima tropical de estação seca no inverno, de acordo com a classificação de Köppen-Geiger (Cardoso et al., 2014).

Os frutos de café foram colhidos de forma manual por meio de coleta por derriça total, onde estes eram arrancados da planta e derriçados no chão coberto por um pano. Os frutos colhidos estavam com teores de água de 1,16 b.s. (base seca, em decimal), sendo necessário a realização de uma pré-secagem. Dessa forma, os frutos de Coffea arabica foram espaIhados em terreiro de concreto e expostos a radiação solar durante 12 horas/dia por 10 dias, iniciando às 06:00 horas e finalizando as 18:00 horas (umidade relativa e temperatura média de $62 \%$ e $23^{\circ} \mathrm{C}$, respectivamente). Os teores de água dos frutos foram reduzidos para 0,11 b.s. (em decimal). Por fim, realizou-se o processo de extração dos grãos, a partir da pilagem dos frutos secos, onde estes apresentavam teores de água de 0,08 b.s. (base seca, em decimal). Os teores de água dos grãos e dos frutos foram determinados por gravimetria utilizando estufa a $105^{\circ} \mathrm{C}$ por $24 \mathrm{~h}$ (ASAE, 2000).

As isotermas de adsorção dos grãos de café arabica foram obtidas utilizando-se o método estático gravimétrico nas temperaturas de $30,40,50$ e $60^{\circ} \mathrm{C}$ e atividade de água (aw) na faixa de 0,10 a 0,83 (decimal) (Tabela 1). Os grãos foram depositados em recipientes de vidro herméticos na qual utilizou-se $36 \mathrm{~g}$ de grãos de Coffea arabica para cada temperatura em estudo, contendo 3 repetições para cada atividade de água $\left(\mathrm{a}_{\mathrm{w}}\right)$. As amostras foram pesadas em balança de precisão de quatro casas decimais, a cada $24 \mathrm{~h}$, até que houvesse uma variação de $0,001 \mathrm{~g}$, representando o equilíbrio higroscópico dos grãos. A temperatura foi controlada por meio de estufa incubadora B.O.D (Demanda Bioquímica de Oxigênio).

Aos dados experimentais, foram ajustados os modelos matemáticos frequentemente utilizados para representação da higroscopicidade de produtos vegetais. Para determinar as propriedades termodinâmicas dos grãos de Coffea arabica, utilizou-se o modelo de Peleg (Tabela 2), que de acordo com Santos et al. (2020), representou adequadamente os dados experimentais, cujo o coeficiente de determinação foi superior a $99,00 \%$ para as temperaturas de $30,40,50$ e $60^{\circ} \mathrm{C}$ e teores de água de 1,91 a $25,34 \%$ (b.s.). Utilizando o modelo foi obtido os valores de atividade de água, pelas equações (Eq.) 1, 2, 3 e 4, para as temperaturas de 30, 40,50 e $60^{\circ} \mathrm{C}$, respectivamente (Tabela 2 ).

A partir da equação de Clausius-Clapeyron (Eq. 5), calculou-se a relação $L^{L^{\prime-1}}$, cujos dados foram ajustados pela equação de Rodrigues-Arias (Eq. 6), e, em seguida, o calor latente de vaporização da água dos grãos (Eq. 7) para cada temperatura e teor de água estudado (Corrêa et al., 1998).

$\operatorname{Ln}(P v)=\frac{L}{L^{\prime}} \operatorname{Ln}(P v s)+C$ 
$\frac{\mathrm{L}}{\mathrm{L}^{\prime}}-1=a \times \exp \left(-b X \mathrm{e}^{\mathrm{m}}\right)$

$L=(2502,2-2,39) T)\left[1+a \times \exp \left(-b \times e^{m}\right)\right]$

em que:

Pv - pressão de vapor de água livre, para determinada temperatura ( $\mathrm{T}$ ) de equilíbrio, $\mathrm{kPa}$;
Pvs - pressão de vapor de saturação da água livre, para determinada temperatura (T) de equilíbrio, kPa;

L - calor latente de vaporização da água do produto, $\mathrm{kJ} \mathrm{kg}^{-1}$;

L' - calor latente de vaporização da água livre, a temperatura de equilíbrio, $\mathrm{KJ} \mathrm{kg}^{-1}$;

C - constante de integração; e,

Tabela 1- Atividade de água (decimal) de soluções salinas nas temperaturas de 30, 40, 50 e 60 C (Labuza et al., 1985)

\begin{tabular}{lccccc}
\hline Temperatura & \multicolumn{5}{c}{ Sal } \\
\cline { 2 - 5 }$\left({ }^{\circ} \mathrm{C}\right)$ & $\mathrm{LiCl}$ & $\mathrm{MgCl}_{2}$ & $\mathrm{Mg}\left(\mathrm{NO}_{3}\right)_{2}$ & $\mathrm{NaCl}$ & $\mathrm{KCl}$ \\
\hline 30 & 0,11 & 0,33 & 0,52 & 0,77 & 0,83 \\
40 & 0,11 & 0,34 & 0,50 & 0,73 & 0,81 \\
50 & 0,10 & 0,30 & 0,48 & 0,71 & 0,77 \\
60 & 0,10 & 0,29 & 0,47 & 0,70 & 0,75 \\
\hline
\end{tabular}

Tabela 2- Equação de Peleg para cálculo da atividade de água (aw) para as temperaturas de $30,40,50$ e $60{ }^{\circ} \mathrm{C}$, para grãos de Coffea arabica.

\begin{tabular}{|c|c|c|}
\hline \multicolumn{3}{|c|}{ Modelo } \\
\hline Temperatura $\left({ }^{\circ} \mathrm{C}\right)$ & $\mathrm{Xe}=\mathrm{k} 1\left(\mathrm{a}_{\mathrm{w}}\right)^{\mathrm{n} 1}+\mathrm{k} 2\left(\mathrm{a}_{\mathrm{w}}\right)^{\mathrm{n} 2}$ & $\mathrm{~N}^{\circ}$ \\
\hline 30 & $X e=0,3320^{* *}\left(a_{w}\right)^{4,4485^{* *}}+0,1106^{* *}\left(a_{w}\right)^{0,2896^{* *}}$ & 1 \\
\hline 40 & $X e=0,6451^{* *}\left(a_{w}\right)^{7,8796^{* *}}+0,1195^{* *}\left(a_{w}\right)^{0,3792^{* *}}$ & 2 \\
\hline 50 & $X e=0,7233^{* *}\left(a_{w}\right)^{6,8747^{* *}}+0,1023^{* *}\left(a_{w}\right)^{0,5687^{* *}}$ & 3 \\
\hline 60 & $X e=1,9819^{* *}\left(a_{w}\right)^{9,6895^{* *}}+0,0802^{* *}\left(a_{w}\right)^{0,5450^{* *}}$ & 4 \\
\hline
\end{tabular}

${ }^{* \star}$ Siginificativo a $5 \%$ pelo teste $\mathrm{t}, \mathrm{Xe}=$ teor de água em decimal (Santos et al., 2020)

A entalpia diferencial, entropia diferencial, teoria da isocinética e energia livre de Gibbs foram calculadas a partir da metodologia descrita por Corrêa et al. (2012), conforme as seguintes equações:

$\ln \left(\mathrm{a}_{\mathrm{w}}\right)=\frac{\Delta \mathrm{h}_{\mathrm{st}}}{\mathrm{RT}}-\frac{\Delta \mathrm{S}}{\mathrm{RT}}$

$\Delta S=\frac{\Delta \mathrm{h}_{\mathrm{st}}-\Delta \mathrm{G}}{\mathrm{T}_{\mathrm{a}}}$

$\Delta \mathrm{h}_{\mathrm{st}}=\mathrm{T}_{\mathrm{b}}(\Delta \mathrm{S})+\Delta \mathrm{G}_{\mathrm{b}}$

$\Delta \mathrm{G}=\mathrm{RT} \mathrm{T}_{\mathrm{a}} \cdot \ln \left(\mathrm{a}_{\mathrm{w}}\right)$

$\mathrm{T}_{\mathrm{hm}}=\frac{\mathrm{n}}{\Sigma\left(\frac{1}{\overline{\mathrm{T}}}\right)}$

$T_{b}=\widehat{T_{b}} \pm t_{m-2, / \alpha \sqrt{\operatorname{Var}\left(T_{b}\right)}}$

em que:

$\Delta \mathrm{h}_{\mathrm{s}}$ - entalpia diferencial de sorção, $\mathrm{kJ} \mathrm{kg}^{-1}$;

$\Delta S$ - entropia diferencial de sorção $\left(\mathrm{kJ} \mathrm{kg}^{-1} \mathrm{~K}^{-1}\right)$;

$\Delta \mathrm{G}$ - energia livre de Gibbs $\left(\mathrm{kJ} \mathrm{kg}^{-1}\right)$;

$\mathrm{T}_{\mathrm{b}}$ - temperatura isocinética $(\mathrm{K})$;

$\Delta G_{B}$ - energia livre de Gibbs à temperatura isocinética $\left(\mathrm{kJ} \mathrm{kg}^{-1}\right)$;

$\mathrm{T}_{\mathrm{hm}}$ - temperatura média harmônica $(\mathrm{K})$; n - número de temperaturas utilizadas;

m - número de pares de dados de entalpia e entropia.

Para análise de regressão não linear, utilizouse o método Gauss-Newton, por meio de o programa computacional Statistica 7.0 ®.

\section{Resultados e discussão}

Os valores de atividade de água estimados pelo modelo de Peleg, paras as temperaturas de 30,40 , 50 e $60^{\circ} \mathrm{C}$, e para os teores de água de equilíbrio entre 1,91 a 25,34\% (b.s.), estão descritos na Tabela 3. Geralmente, com o aumento do teor de água de equilíbrio ocorre o aumento da atividade de água (Silva et al., 2018). Dessa forma, verifica-se que tal efeito ocorre no presente trabalho, onde nas temperaturas de $30,40,50^{\circ} \mathrm{C}$ e $60^{\circ} \mathrm{C}$ o aumento do teor de água promove o aumento da atividade de água. Em relação ao aumento de temperatura, verifica-se que a atividade de água tende a aumentar até o teor de água de $13,52 \%$, nas temperaturas de 30,40 e $50^{\circ} \mathrm{C}$. Já para a temperatura de $60^{\circ} \mathrm{C}$, verifica-se que a atividade de água foi menor, quando comparado com as demais. Isso pode ser explicado devido às características higroscópicas do produto, onde as isotermas de adsorção de Coffea arabica foram praticamente independentes da temperatura em altos valores de atividades de água (Santos et al., 2020). Resultados seme- 
Ihantes foram encontrados por Sandoval \& Barreiro (2002), que trabalharam com isotermas de sorção de água de grãos de cacau não fermentados. Além disso, este comportamento tem sido reportado na literatura para níveis de atividade de água e temperaturas diferentes, dependendo do teor de açúcares do produto (Saravacos et al., 1986; Tsami et al.,1990).

A relação $L^{\prime \prime-1}$ (relação entre o calor latente de vaporização da água do produto e calor latente de vaporização da água livre ) apresentou valores maiores ou próximos de 1 para toda a faixa de teor de água de equilíbrio estudada (Figura 1), evidenciando que a energia liberada pelos grãos durante o processo de adsorção é semelhante ou maior que o calor latente de vaporização da água livre $\left(\mathrm{L}^{\prime}=2.454,400 \mathrm{~kJ} \mathrm{~kg}^{-1}\right)$ (Silva et al., 2018), fato também observado em grãos de milho (Smaniotto et al., 2012) e em sementes de pimenta malagueta (Silva \& Rodovalho, 2015). Percebe-se, também, que a relação $\mathrm{LL}^{\prime-1}$ é inversamente proporcional ao teor de água dos grãos de Coffea arabica, ou seja, quanto maior a relação $\mathrm{LL}^{-1}$, menor será o teor de água nos grãos.

Os parâmetros a b e m da Eq. (6), para o cálculo da razão entre o calor latente de vaporização dos grãos de Coffea arabica e o vapor latente de vaporização de água livre $\left(\mathrm{LL}^{-1}\right)$, foram obtidos por meio de regressão não linear e, posteriormente, a Eq. (7) foi utilizada para calcular os valores de cada calor latente de vaporização da água de acordo com a temperatura (Figura 2). Â medida que o teor de água aumenta, há uma diminuição na energia necessária para a evaporação da água do produto (Sousa et al., 2016), na qual, constata-se que para as temperaturas de 30, 40, 50 e $60{ }^{\circ} \mathrm{C}$ e teor de água de equilíbrio de 1,91 a $25,34 \%$ (b.s.), o calor latente de vaporização variou de $8.334,62$ a $2.358,87 \mathrm{~kJ} \mathrm{~kg}^{-1}$.

Tabela 3 - Valores da atividade da água (decimal) estimados pelo modelo Peleg em função da temperatura e do teor de água de equilíbrio higroscópico dos grãos de Coffea arabica.

\begin{tabular}{|c|c|c|c|c|c|c|c|c|c|}
\hline \multirow{2}{*}{ Xe (\%b.s.) } & \multicolumn{4}{|c|}{ Temperatura $\left({ }^{\circ} \mathrm{C}\right)$} & & \multicolumn{4}{|c|}{ Temperatura $\left({ }^{\circ} \mathrm{C}\right)$} \\
\hline & $30^{\circ} \mathrm{C}$ & $40^{\circ} \mathrm{C}$ & $50^{\circ} \mathrm{C}$ & $60^{\circ} \mathrm{C}$ & & $30^{\circ} \mathrm{C}$ & $40^{\circ} \mathrm{C}$ & $50^{\circ} \mathrm{C}$ & $60^{\circ} \mathrm{C}$ \\
\hline 1,91 & 0,007 & 0,051 & 0,069 & 0,002 & 13,52 & 0,682 & 0,684 & 0,706 & 0,616 \\
\hline 1,95 & 0,009 & 0,053 & 0,071 & 0,002 & 13,82 & 0,689 & 0,688 & 0,710 & 0,626 \\
\hline 2,27 & 0,012 & 0,068 & 0,093 & 0,004 & 15,84 & 0,727 & 0,717 & 0,727 & 0,679 \\
\hline 2,58 & 0,016 & 0,085 & 0,116 & 0,006 & 15,86 & 0,728 & 0,717 & 0,727 & 0,679 \\
\hline 2,81 & 0,020 & 0,098 & 0,144 & 0,009 & 15,96 & 0,730 & 0,718 & 0,728 & 0,681 \\
\hline 2,83 & 0,020 & 0,099 & 0,147 & 0,025 & 16,19 & 0,733 & 0,720 & 0,730 & 0,687 \\
\hline 3,89 & 0,051 & 0,181 & 0,262 & 0,025 & 16,26 & 0,734 & 0,721 & 0,731 & 0,688 \\
\hline 4,49 & 0,074 & 0,2335 & 0,339 & 0,043 & 16,45 & 0,737 & 0,724 & 0,732 & 0,692 \\
\hline 4,96 & 0,095 & 0,277 & 0,404 & 0,060 & 18,43 & 0,761 & 0,747 & 0,745 & 0,734 \\
\hline 5,02 & 0,098 & 0,282 & 0,411 & 0,062 & 19,13 & 0,771 & 0,754 & 0,750 & 0,745 \\
\hline 5,02 & 0,098 & 0,282 & 0,412 & 0,062 & 19,97 & 0,779 & 0,763 & 0,755 & 0,759 \\
\hline 5,04 & 0,099 & 0,284 & 0,414 & 0,063 & 20,62 & 0,786 & 0,768 & 0,758 & 0,769 \\
\hline 5,10 & 0,102 & 0,290 & 0,423 & 0,065 & 20,62 & 0,786 & 0,768 & 0,758 & 0,769 \\
\hline 5,12 & 0,103 & 0,292 & 0,425 & 0,066 & 20,69 & 0,787 & 0,768 & 0,759 & 0,770 \\
\hline 5,12 & 0,103 & 0,292 & 0,426 & 0,066 & 21,24 & 0,791 & 0,773 & 0,762 & 0,778 \\
\hline 5,35 & 0,115 & 0,315 & 0,459 & 0,076 & 21,73 & 0,796 & 0,777 & 0,765 & 0,785 \\
\hline 5,42 & 0,119 & 0,322 & 0,469 & 0,084 & 21,84 & 0,797 & 0,778 & 0,765 & 0,788 \\
\hline 5,66 & 0,138 & 0,345 & 0,500 & 0,097 & 22,50 & 0,802 & 0,783 & 0,768 & 0,797 \\
\hline 5,84 & 0,150 & 0,363 & 0,506 & 0,109 & 22,73 & 0,805 & 0,785 & 0,770 & 0,800 \\
\hline 5,85 & 0,151 & 0,365 & 0,507 & 0,109 & 23,02 & 0,807 & 0,787 & 0,771 & 0,804 \\
\hline 5,87 & 0,152 & 0,366 & 0,508 & 0,110 & 24,69 & 0,820 & 0,800 & 0,779 & 0,826 \\
\hline 7,26 & 0,265 & 0,490 & 0,591 & 0,221 & 24,87 & 0,821 & 0,802 & 0,780 & 0,828 \\
\hline 7,30 & 0,269 & 0,492 & 0,592 & 0,224 & 25,34 & 0,825 & 0,805 & 0,782 & 0,834 \\
\hline 7,32 & 0,271 & 0,493 & 0,592 & 0,226 & & & & & \\
\hline 7,73 & 0,311 & 0,509 & 0,608 & 0,267 & & & & & \\
\hline 7,82 & 0,321 & 0,519 & 0,612 & 0,277 & & & & & \\
\hline 7,84 & 0,322 & 0,520 & 0,613 & 0,278 & & & & & \\
\hline 8,24 & 0,366 & 0,538 & 0,625 & 0,320 & & & & & \\
\hline 8,33 & 0,376 & 0,542 & 0,627 & 0,330 & & & & & \\
\hline 8,38 & 0,381 & 0,544 & 0,628 & 0,335 & & & & & \\
\hline 9,44 & 0,502 & 0,587 & 0,651 & 0,427 & & & & & \\
\hline 9,46 & 0,504 & 0,588 & 0,651 & 0,429 & & & & & \\
\hline 9,48 & 0,507 & 0,589 & 0,651 & 0,430 & & & & & \\
\hline 11,00 & 0,599 & 0,631 & 0,675 & 0,520 & & & & & \\
\hline 11,04 & 0,601 & 0,632 & 0,676 & 0,522 & & & & & \\
\hline 11,15 & 0,608 & 0,635 & 0,678 & 0,522 & & & & & \\
\hline 12,09 & 0,641 & 0,656 & 0,691 & 0,566 & & & & & \\
\hline
\end{tabular}




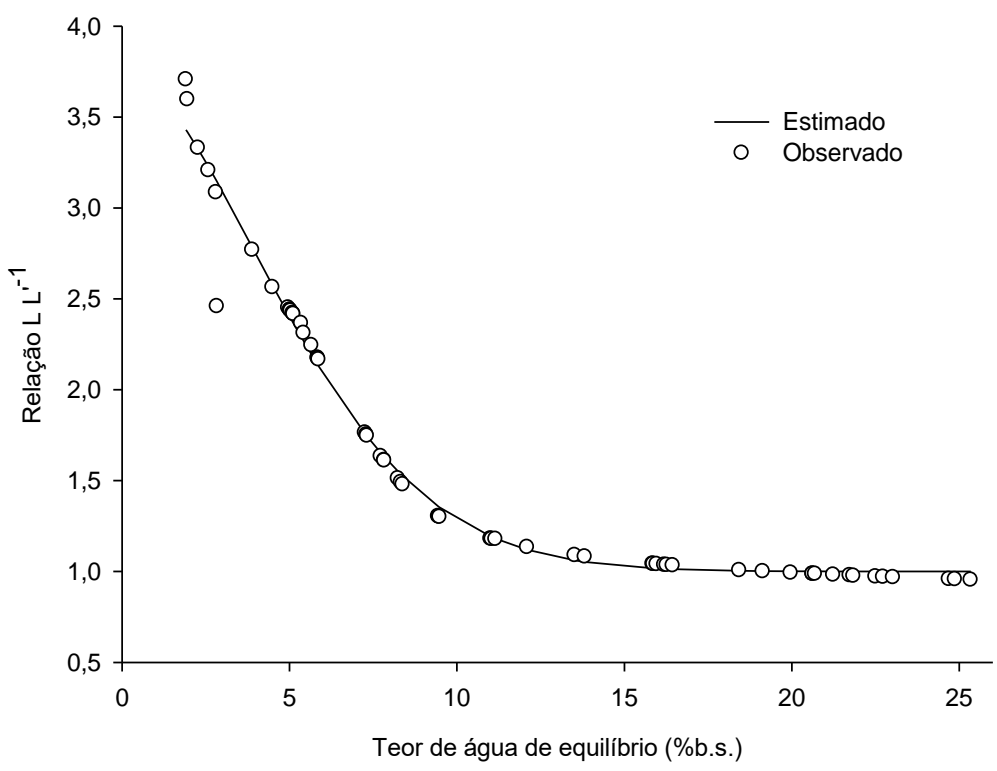

Figura 1 - Relação L L’-1 em função do teor de água de equilíbrio em grãos de Coffea arabica.

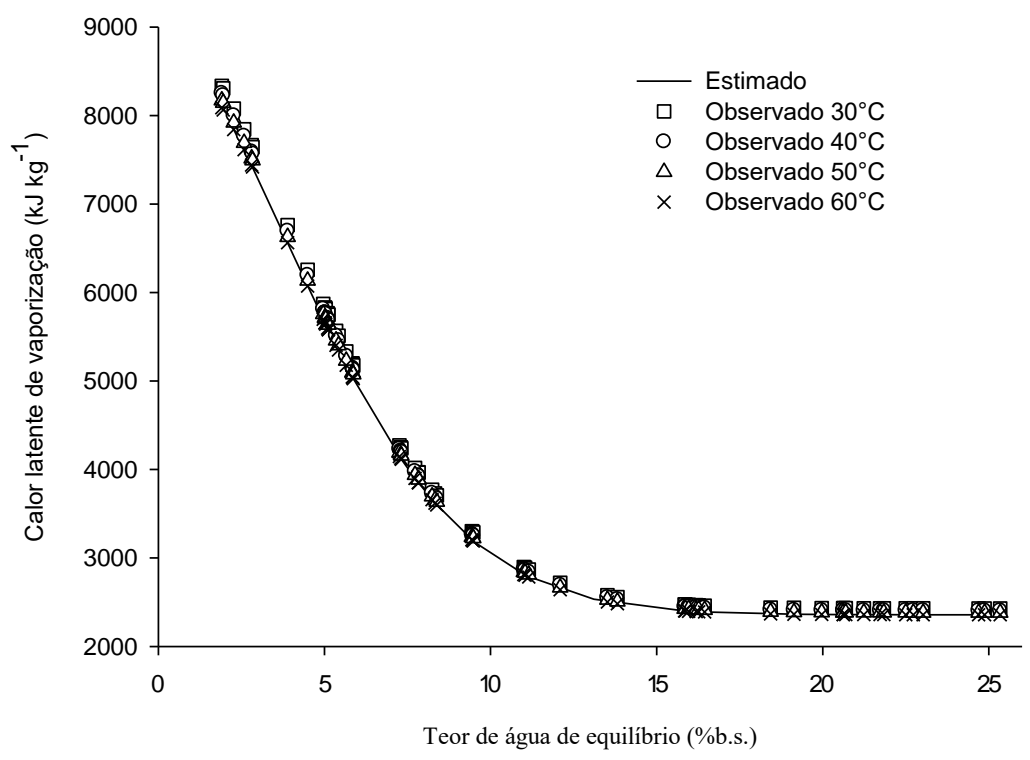

Figura 2 - Calor latente de vaporização de adsorção em função do teor de água de equilíbrio em grãos de Coffea arabica.

Verifica-se na Figura 3, que a redução do teor de água dos grãos de Coffea arabica promove o aumento da entropia diferencial. Tal efeito ocorre devido ao aumento do grau de desordem das moléculas de água contidas no interior dos grãos (Majd et al., 2013). Além disso, com elevados valores de atividade de água, e consequentemente, maiores teores de água, há menos locais disponíveis para as ligações entre as moléculas, implicando em menor demanda de mobilidade das moléculas de água (Goneli et al., 2010).
Os valores de entropia diferencial de grãos Coffea arabica variam de $-0,09$ a $3,37 \mathrm{~kJ} \mathrm{~kg}^{-1} \mathrm{~K}^{-1}$. Os valores negativos de entropia diferencial podem estar atribuídos à existência de adsorção química e/ou modificações estruturais do adsorvente (Moreira et al., 2008). Zeymer et al. (2020), que trabalharam com sementes de ryegrass (Lolium multiflorum L.) verificaram o mesmo comportamento que o presente trabalho ( 0.03 a $\left.4.45 \mathrm{~kJ} \mathrm{~kg}^{-1} \mathrm{~K}^{-1}\right)$ como também Goneli et al. (2010) que trabalharam com sementes de 
Abelmoschus esculentus $\mathrm{L}\left(0,10\right.$ to $\left.10,84 \mathrm{~kJ} \mathrm{~kg}^{-1} \mathrm{~K}^{-1}\right)$.

A representação da relação entalpia e entropia, no processo de adsorção, para os grãos de Coffea arabica, foi ajustada segundo uma regressão linear, apresentando elevado coeficiente de determinação $(0,9430)$ (Figura 4), indicando a existência da teoria isocinética para o processo de adsorção. Na compensação linear entalpia-entropia, deve-se comparar os valores $T_{b}$ (temperatura isocinética) e $T_{h m}$ (Temperatura média harmônica). Se $T_{b}>T_{h m}$, o processo é controlado pela entalpia; se $T_{b}<T_{h m}$, o processo é controlado pela entropia (Krug et al., 1976a; b).
Desta maneira, a $T_{b}$ obtida foi de $498,3433 \pm 5,7013 \mathrm{~K}$, enquantoà $\mathrm{T}_{\mathrm{hm}}$ foi de $317,7568 \mathrm{~K}$, confirmando assim o fenômeno da teoria da isocinética para o processo de adsorção. Neste contexto, confirma-se ainda, que a $T_{b}$ foi maior que a $T_{h m}$, evidenciando que o processo é controlado pela entalpia. Estes resultados estão de acordo com o que foi observado por diversos pesquisadores, que têm aplicado, com sucesso, a teoria isocinética sobre a sorção de diversos produtos agrícolas (Goneli et al., 2010, Oliveira et al., 2013, Rodrigues et al., 2020).

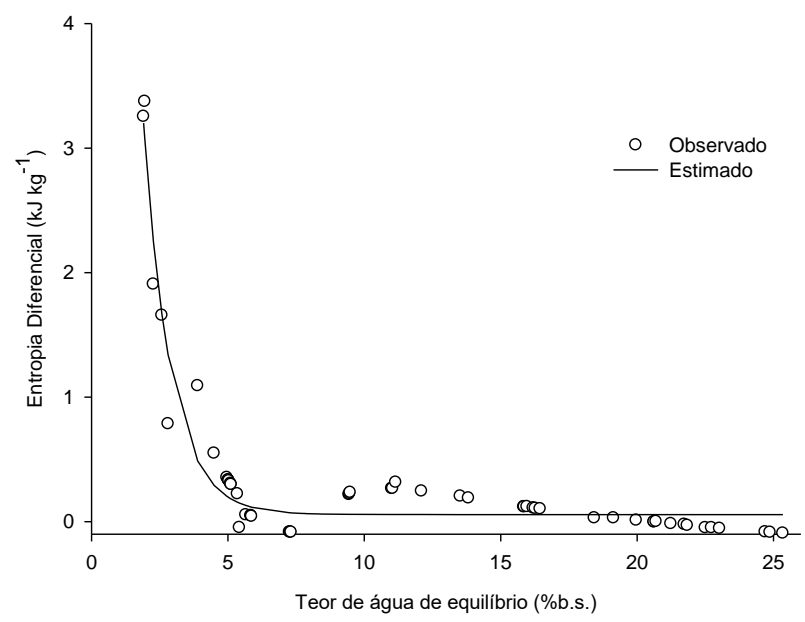

Figura 3 - Valores experimentais e estimados de entropia diferencial de adsorção em função do teor de água de equilíbrio em grãos de Coffea arabica.

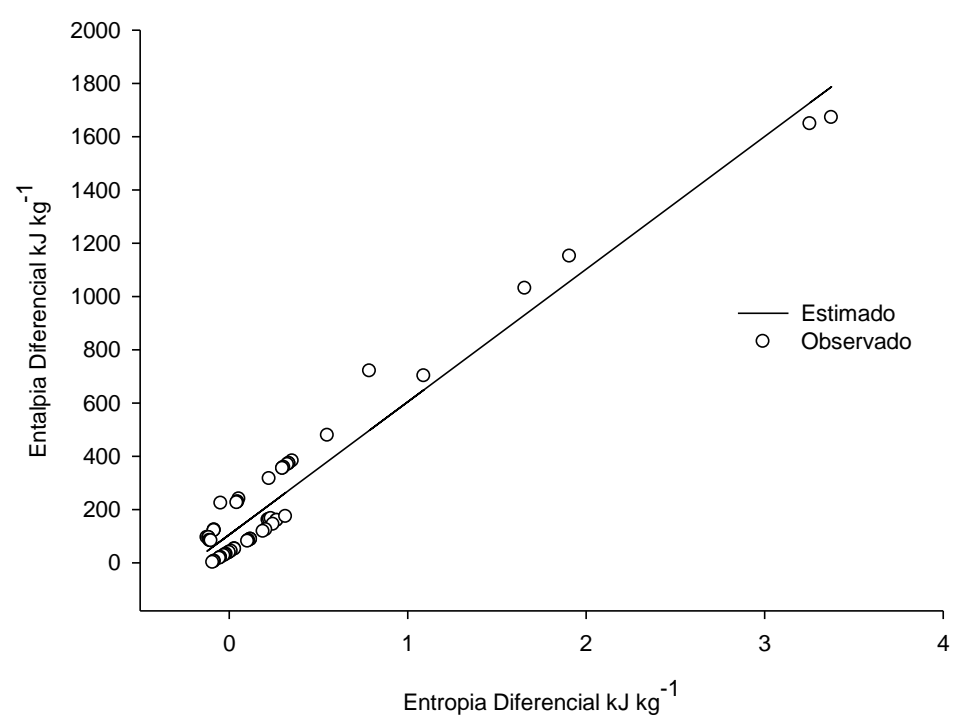

Figura 4 - Relação linear entalpia-entropia para o processo de adsorção em função do teor de água de equilíbrio em grãos de Coffea arabica.

$\mathrm{Na}$ Figura 5, verifica-se que a energia livre de Gibbs para os grãos de Coffea arabica, com teor de água variando de 25,34 a $1,31 \%$ (b.s.) e temperaturas de $30,40,50$ e $60^{\circ} \mathrm{C}$, variou entre 26,86 para $959,48 \mathrm{~kJ} \mathrm{~kg}^{-1}$. Além disso, esta propriedade aumentou com o decréscimo do teor de água, sendo positiva para 
todas as temperaturas estudadas, com tendência a se estabilizar. Tal fato foi verificado por Oliveira et al. (2013) para grãos de milho e por Thys et al. (2010) para sementes de pinhão.

Ainda na Figura 5, para as temperaturas de 30, 40 e $50^{\circ} \mathrm{C}$, constata-se que a energia livre de Gibbs diminui com o aumento da temperatura. Isso pode ser explicado pelo maior grau de movimento de moléculas acelerando a troca de massa, acelerando assim o processo em níveis mais elevados de teor de água de equilíbrio. Todavia, para a temperatura de $60^{\circ} \mathrm{C}$, verifica-se que esta propriedade teve um comportamento atípico, apresentado valores mais elevados do que as demais temperaturas. Este fato está relacionado com a constituição química dos grãos, sendo um fator dependente dos teores de açúcares (Saravacos et al., 1986; Tsami et al.,1990) e, também, a possíveis erros na obtenção dos valores de atividade de água (principalmente em temperaturas elevadas), uma vez que os valores foram obtidos a partir de modelo matemático (Ferreira \& Pena, 2003).

Valores positivos para energia livre de Gibbs caracterizam uma reação endotérmica (Silva et al., 2016), ou seja, aquela que necessita de energia para que ocorra a sorção da água. Portanto, para que ocorra a redução do teor de teor de água de equilíbrio dos grãos de Coffea arabica é necessário o fornecimento de energia para o sistema, caracterizando assim um processo não espontâneo.

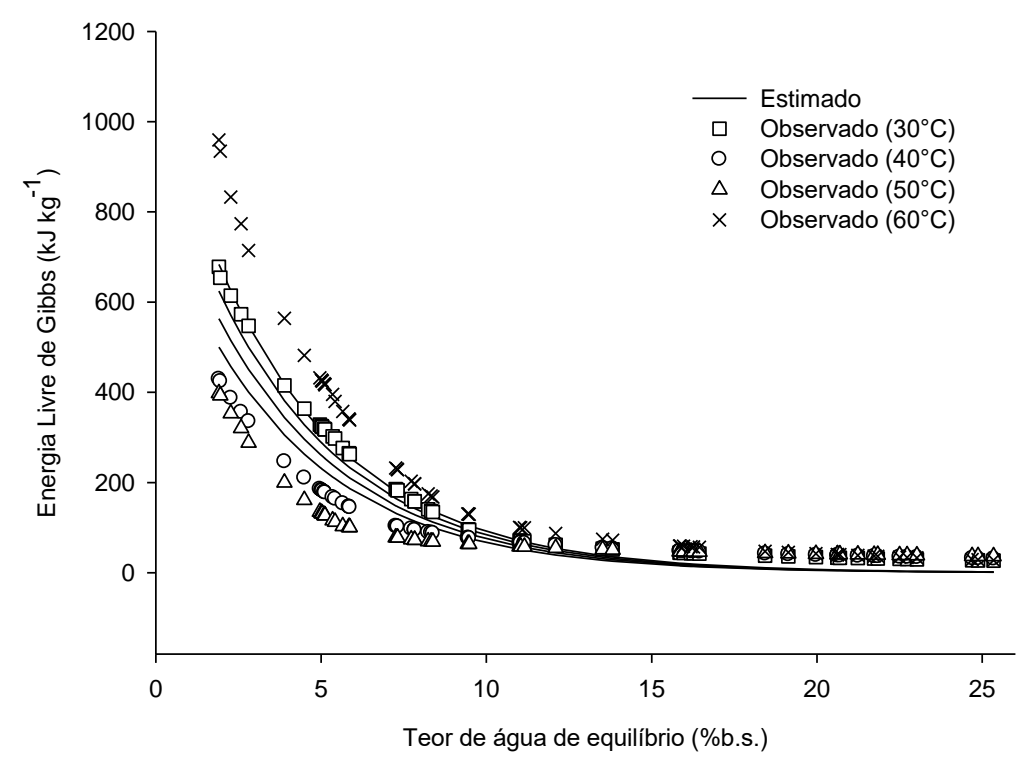

Figura 5 - Valores experimentais e estimados de energia livre de Gibbs em função da temperatura e teor de água de equilíbrio em grãos de Coffea arabica.

$\mathrm{Na}$ Tabela 4, verifica-se as equações de regressão ajustadas à relação $L L^{\prime-1}$, entropia diferencial, relação entalpia-entropia diferencial e energia livre de Gibbs de adsorção. Estas equações podem ser utilizadas para estimar estes parâmetros termodinâmicos para o processo de adsorção de grãos de Coffea arabica para o intervalo de teor de água de equilíbrio na faixa de 25,34 a 1,31\% (b.s.) e temperaturas de 30, 40, 50 e $60^{\circ} \mathrm{C}$.

Tabela 4- Equações de regressão e coeficientes de determinação para a relação $L L^{\prime-1},(\Delta S)$, relação entalpiaentropia e energia livre d Gibbs $(\Delta \mathrm{G})$ de adsorção em função do teor de água de equilíbrio de grão de Coffea arabica.

\begin{tabular}{lc}
\hline Equação de regressão & $\mathrm{R}^{2}$ \\
\hline $\mathrm{L} \mathrm{L}^{\prime-1}=1+2,7572^{* *} \exp \left(-123,9317^{* *} \mathrm{Xe}^{1,7402^{* *}}\right)$ & 0,9818 \\
$\Delta \mathrm{S}=-0,0572^{* *}+21,4585^{* *} \exp \left(-1,0046^{* *} \mathrm{Xe}\right)$ & 0,9321 \\
$\Delta \mathrm{H}_{\mathrm{st}}=4,98,3434^{* *} \Delta \mathrm{S}-\left(-105,9600^{* *}\right)$ & 0,9430 \\
$\Delta \mathrm{G}=\left(-3128,4195^{* *} \ln (\mathrm{T})+(-17070,6763)^{* *}\right) \exp \left(-0,2492^{* *} \mathrm{Xe}\right)$ & 0,7654 \\
\hline
\end{tabular}

\footnotetext{
${ }^{* *}$ Significativo pelo teste $t(p<0,05)$.
} 


\section{Conclusões}

As propriedades termodinâmicas foram influenciadas pelo teor de água dos grãos de Coffea arabica, onde foi necessário o aumento da energia para retirara de água do produto em teores de água mais baixos.

O calor latente de vaporização, a entropia diferencial e a energia livre de Gibbs aumentam com a redução do teor de água de equilíbrio dos grãos.

O fenômeno da compensação entalpia-entropia é válido para o processo de adsorção dos grãos de Coffea arabica, sendo este controlado pela entalpia.

\section{Agradecimentos}

Esta pesquisa foi apoiada pelo Instituto Federal Goiano (Campus Ceres), Fundação de Amparo à Pesquisa do Estado de Goiás (FAPEG) e Conselho Nacional de Desenvolvimento Científico e Tecnológico (CNPq).

\section{Referências}

ABIC (2018) Associação Brasileira da Indústria de Café Estatísticas - indicadores da indústria de café no Brasil. Disponível https://www.abic.com.br/estatisticas/indicadores-daindustria/indicadores-da-industria-de-cafe-2018-2/> . (Acesso em: 16 de setembro de 2020).

ASAE- American Society of Agricultural Engineers (2000) Moisture measurement-ungrounded grains and seeds, St. Joseph:, 563p.

Araújo LF Corrêa PC, Silva RF (2001) Comparação de modelos matemáticos para descrição das curvas de dessorção de sementes de milho-doce. Pesquisa Agropecuária Brasileira 36(7):991-995.

Bakhtavar MA, Afzal I, Basra SMA (2019) Moisture adsorption isotherms and quality of seeds stored in conventional packaging materials and hermetic super bag. Plos One 14(2): 1-11.

Cardoso MRD, Marcuzzo FFN, Barros JB (2014) Classificação climática de Köpper-Geiger para o estado de Goiás e o Distrito Federal. Acta Geográfica 8(16): 40-55.

Corrêa PC, Botelho FM, Botelho SCC, Goneli ALD (2014) Isotermas de sorção de água de frutos de Coffea canephora. Revista Brasileira de Engenharia Agrícola e Ambiental 18(10): 1047-1052.

Corrêa PC, Christ D, Martins JH, Mantovani BHM (1998) Curvas de dessorção e calor latente de vaporização para as sementes de milho pipoca (Zea mays). Revista Brasileira de Engenharia Agrícola e Ambiental 2(1): 75-79.

Corrêa PC, Oliveira GHHD, Santos EDS (2012) Thermodynamic properties of agricultural products processes. Physical properties of foods: Novel measurement techniques and applications. Chap.6: 131-141.
Costa LM, Resende O, Oliveira DECD, Sousa KA (2015) Isotherms and isosteric heat in the seeds of Buchenavia capitata (Vahl) Eichler. Revista Ciência Agronômica 46(3): 516-523.

Ferreira CD, Pena RS (2003) Comportamento higroscópico da farinha de pupunha (Bactris gasipaes). Ciência e Tecnologia de Alimentos 23(2): 251-255.

Ferreira Junior WN, Resende O, Oliveira DECD, Costa LM (2018) Isotherms and Isosteric Heat Desorption of Hymenaea stigonocarpa Mart. Seeds. Journal Of Agricultural Science 10(10): 504-512.

Goneli ALD, Corrêa PC, Oliveira GHH, Botelho FM (2010) Water desorption and thermodynamic properties of okra seeds. Transaction of the ASAE 53(1): 191-197.

Kaya S, Kahyaoglu T (2006) Influence of dehulling and roasting process on the thermodynamics of moisture adsorption in sesame seed. Journal of Food Engineering 76(2): 139-147.

Krug RR, Hunter WG, Grieger RA (1976a) Enthalpyentropy compensation: 1 - Some fundamental statistical problems associated with the analysis of van't Hoff and Arrhenius data. Journal of Physical Chemistry 80(1): 2335-2341.

Krug RR, Hunter WG, Grieger RA (1976b) Enthalpyentropy compensation: 2 - Separation of the chemical from the statistical effect. Journal of Physical Chemistry 80(1): 2341-2351.

Labuza TP, Kaauane A, Chen JY (1985) Effect of temperature on the moisture sorpion isotherms and water activity shift of two dehydrated foods. Journal of Food Science 50(1): 385-391.

Majd KM, Karparvarfard SH, Farahnaky A, Jafarpour K (2013) Temperature effect of sorption isotherms and thermodynamic characteristics. Food Biophysics, 8(1): 1-11.

Moreira R, Chenlo F, Torres MD, Vallejo N (2008) Thermodynamic analysis of experimental sorption isotherms of loquat and quince fruits. Journal of Food Engineering 88(1): 514-521.

Oliveira DECD, Resende O, Chaves TH, Souza KAD, Smaniotto TADS (2014) Propriedades termodinâmicas das sementes de pinhão-manso. Bioscience Journal 30(1): 147-157.

Oliveira DECD, Resende O, Smaniotto TADS, Sousa KAD, Campos RC (2013) Propriedades termodinâmicas de grãos de milho para diferentes teores de água de equilíbrio. Pesquisa Agropecuária Tropical 43(1): 5056.

Rodrigues KC, Silva HWD, Silva IL, Santos SGFD, Silva DPD, Rodovalho RS (2020) Isotherms and thermodynamic properties of water adsorption in 'Cumari-do-Pará' pepper seeds. Revista Brasileira de Engenharia Agrícola e Ambiental 24(4): 280-285. 
Tsami E, Marinos-Kouris D, Maroulis ZB (1990) Water sorption isotherms of raisins, currants, figs, prunes and apricots. Journal of Food Science 55(6):1594-1597.

Sandoval AJ, Barreiro JA (2002) Water sorption isotherms of non-ermented cocoa beans (Theobroma cacao). Journal of Food Engineering 51(1): 119-123.

Santos SGFD, Silva DPD, Sarti JK, Almeida VG, Rodovalho RS (2020) Isotermas de adsorção e calor isostérico em grãos de Coffea arabica. Pesquisa Agropecuária Tropical 50(1): 1-7.

Saravacos GD, Tsiourvas DA, Tsami E (1986) Effect of temperature no the water adsorption isotherms of sultana raisins. Journal of Food Science 51(2):381-383.

Silva HWD, Costa LM, Resende O, Oliveira DECD, Soares RS, Vale LS (2016) Thermodynamic properties of pepper seeds -Variety 'Cabacinha'. Científica 44(1): 14-22.

Silva HWD, Oliveira DECD, Resende O, Costa LM (2018) Thermodynamic properties of water desorption in Buchenavia capitata (Vahl) Eichler. Revista Brasileira de Engenharia Agrícola e Ambiental 22(12): 878-883.
Silva HWD, Rodovalho RS (2015) Adsorption isotherms and vaporization latent heat of Malagueta pepper seeds. Científica 44(1): 5-13.

Smaniotto TAS, Resende O, Oliveira DEC, Sousa KA, Campos RC (2012) Isotermas e calor latente de dessorção dos grãos de milho da cultivar AG 7088. Revista Brasileira de Milho e Sorgo 11(3): 312-322.

Sousa KAD, Resende O, Carvalho BDS (2016) Determination of desorption isotherms, latent heat and isosteric heat of pequi diaspore. Revista Brasileira de Engenharia Agrícola e Ambiental 20(5): 493-498.

Thys RCS, Noreña CPZ, Marczaka LDF, Aires AG, Cladera-Olivera $F$ (2010) Adsorption isotherms of pinhão (Araucaria angustifólia seeds) starch and thermodynamic analysis. Journal of Food Engineering 100(3): 468-473.

Zeymer JS, Corrêa PC, Oliveira GHHD, Araujo MEVD, Magalhães DS (2020) Thermodynamic properties of moisture desorption isotherms of ryegrass (Lolium multiflorum L.) seeds. Ciência e Agrotecnologia 44(1): 1-8. 